Modern Physics Letters B

(C) World Scientific Publishing Company

\title{
ERRATA
}

\section{METASTABLE STATES AND IRREGULAR BEHAVIOUR IN A JOSEPHSON JUNCTION WITH NONLINEAR RESISTANCE}

[MOD. PHYS. LETT. B, Vol. 5, No. 7 (1991) 519-529]

G. AMBIKA, K. BABU JOSEPH, and V. M. NANDAKUMARAN

Sections 3 and 4 should read as follows:

\section{Profiles of Motion and Response Modes}

The dynamics of the junction described by (3) can be simulated by a particle moving dissipatively in a potential $U(\theta)$ given by

$$
U(\theta)=1-\left(\cos \theta+\rho_{1} \theta \sin \omega t+\rho_{0} \theta\right) .
$$

This corresponds to a series of potential wells whose barrier heights are modified by the biasing parameters $\rho_{1}$ and $\rho_{0}$. The central well has $-\pi / 2<\theta<\pi / 2$ with the minimum at $\theta=0$. The motion of the system in general is of the oscillatory type confined to the central well for low values of $\rho_{1}$, while for large values of $\rho_{1}$ the system escapes from the well and describes running modes over the potential maxima. We find that the response of the system, both before and after the escape, depends primarily on the frequency of the driving current $\omega$. Depending on the value of $k$, after escaping from the well, the system may get itself trapped in any of the successive minima too. Chaotic states arise due to random shuttling between running and oscillatory modes.

For our analysis, (3) is rewritten as a system of three first order equations. With $\theta=x_{1}$ we have 


$$
\begin{aligned}
& \dot{x}_{1}=x_{2}, \\
& \dot{x}_{2}=-k\left|x_{2}\right| x_{2}-\sin x_{1}+\rho_{0}+\rho_{1} \sin x_{3}, \\
& \dot{x}_{3}=\omega .
\end{aligned}
$$

This constitutes a three dimensional dissipative system with quadratic damping. We restrict the analysis to the case of extreme underdamping with $k=0.1$. For low values of $\omega$, namely, $0.02<\omega<0.2$, the system behaves adiabatically and the profile of motion has structure on a very fine scale. The trajectory shows a screw type motion around the $\theta$ axis (Fig. 1a) which is being repeated as an oscillation over a certain region with the periodicity of the applied force. The amplitude of the motion decreases after each oscillation but the system refuses to settle down to a stable trajectory. The pitch of this screw type motion increases with $\rho_{1}$ until for a critical value $\rho_{c}$ the trajectory escapes from the central well. The junction voltage in this frequency region shows a square wave pattern, which under finer scaling reveals periodic motions in two widely differing time scales (Fig. 1b).

\section{Metastable State}

In the frequency range $0.2<\omega<1$, we observe that the system exhibits a metastable oscillatory mode characterised by a trajectory that continues indefinitely for a long interval of time (Fig. 2a). The continuous disturbance introduced by the quadratic damping prevents the system from forming stable limit cycles. The small fluctuations associated with the transient nature of the motion leads to broading of peaks in the power spectrum of voltage. This is clear from Fig. 2b where the Fast Fourier Transform (FFT) ${ }^{12}$ of the junction voltage is given. The corresponding Poincare map shows a very slowly advancing curve. A quantitative characterisation of this metastable state, we feel, is possible through the Lyapunov Characteristic Exponent (LCE), which measures the average rate of convergence or divergence of nearby trajectories. Using the conventional techniques, ${ }^{13}$ we compute the maximum LCE $\lambda_{\max }$ and find that for the metastable state the value of $\lambda_{\max }$ is very small, $\approx 10^{-2}$. Whether it is positive or negative depends, in this context, on $\omega$. Metastable states can thus decay into a stable trajectory or develop into a chaotic attractor, after a sufficiently long interval of time. If $\lambda_{\max }$ is positive, the ultimate state is chaotic while a negative $\lambda_{\max }$ takes the trajectory asymptotically to stable limit cycle. Here the rate of convergence or divergence is seen to follow a power law rather than an exponential behaviour that is usually observed near limit cycles or chaotic attractors.

The transient nature of the solution to (3) is clear from the last term in (6). What the external periodic force does is to prevent the transients from dying down fast and thus sustain them, thereby resulting in metastability. This is especially so for low damping which we consider here. The fluctuations in the junction voltage corresponding to this state is given in Fig. 2c. As $\omega$ is increased further, the 


\section{Errata 1039}

metastable nature of the trajectory prevails, but $\lambda_{\max }$ becomes positive, though small. 